Contents available at PubMed

Rev Inves Clin. 2018;70:40-45

\title{
ChANGING TRENDS IN GASTRIC POLYPS
}

\author{
Magali Evangelina VelázQuez-Dohorn, Carlos Fernando López-Durand and \\ ARMANDO GAMBOA-DOMÍNGUEZ
}

Department of Pathology, Instituto Nacional de Ciencias Médicas y Nutrición Salvador Zubirán, Mexico City, Mexico

\begin{abstract}
\end{abstract}
Background: The prevalence of gastric polyps varies around the world reflecting regional associations. We describe dem@graphic features of patients with gastric polyp diagnosis treated between 1980 and 2016 at a referral center in Mexico City and analyzed trends of polyp subtype. Materials and Methods: We conducted a blind review of archival slides of gastric biopsies with polyp diagnosis from the years 1980, 1990, 2000, 2010, and 2016. Initial diagnosis; patient's gender, age and symptoms; and number and location of lesions were recorded. Blind slide review and trend analysis were performed. Results: In 3887 gastric biopsies, 192 patients (4.93\%) with epithelial polyps were identified. The median age of patients was 58 years; $73 \%$ were femalę Polyps were single in $143 / 192$ cases $(74.4 \%)$, almost $67 \%$ in the oxyntic mucosa, and $85 \%$ were associated with dyspepsia. The prevalence was $0.5 \%, 1.6 \%, 1.9 \%, 4.6 \%$, and $9.6 \%$ for the years $1980,1990,2000,2010$, and 2016 , respectively, resulting in a rising trend in the prevalence of epithelial polyps of $380 \%$ in 46 years. Fundic gland polyps (FGPs) had a global freqüency of $66.6 \%(128 / 192)$. They were identified for the first time in the third period of the study, with a frequency of $28.6 \% \overline{86} / 21$ ), $66.6 \%$ (35/53), and 78.3\% (87/111) for the years 2000, 2010, and 2016, respectively. Contrary, hyperplastic polyps (HPs) decreased $20 \%$. A relative prevalence of $3.29 \%, 0.97 \%$, and $0.15 \%$ was observed for FGP, HP, and gastric adenoma, respectively. Discussion: The $1400 \%$ change of FGP explains the increased prevalence of gastric polyps. Chronic treatment with proton pump inhibitors and Helicobacter pylori eradication are possible explanations.

Key words: Gastric polyp. Time trend. Prevalence. Fundic gland polyp. Hyperplastic polyp. Gastric adenoma. Mexico.

\section{INTRODUCTION}

A polyp is any protrusion from the surface of the gastrointestinal mucosa observed with the endoscope. These lesions may be heterotopias, lymphoid tissue, stromal lesions, or true epithelial polyps. Differences in histology, gross aspect, and environmental and clinical associations are observed between hyperplastic, fundic gland, and adenomatous polyps, the three most frequently recognized subtypes of gastric polyps. Histologic diagnosis of polyp subtype is necessary in

\footnotetext{
Corresponding author:

*Armando Gamboa-Domínguez

Vasco de Quiroga, 15

Col. Belisario Domínguez Sección XVI, Del. Tlalpan

C.P. 14080, Mexico City, Mexico

E-mail: agamboad@gmail.com
}

endoscopies with suspicious results for the adequate treatment and follow-up of patients. Approximately $20 \%$ of biopsy specimens identified endoscopically as polyps have no definitive pathological diagnosis్

Hyperplastic foveolae with cystic gland dilation and a variable amount of inflamed stroma in the antrum are the typical picture of hyperplastic polyps (HP); the surrounding mucosa depicts chronic gastritis with Helicobacter pylori infection, and varying foci of intestinal metaplasia or dysplasia can be observed. Nearly
Received for publication: 16-10-2017

Approved for publication: 30-11-2017

doi: $10.24875 /$ RIC. 17002430 
$<1 \%$ of hyperplastic lesions can contain adenocarcinoma within the polyp, but it can be up to $6 \%$ in the adjacent gastric mucosa ${ }^{2}$. Fundic gland polyps (FGPs) are located in the oxyntic mucosa and associated with a chronic use of proton pump inhibitors (PPIs). Parietal cell hyperplasia and protrusion to cystic glandular spaces with edema and minimal inflammation in the stroma are prototypical findings ${ }^{3}$. Chronic inflammation in gastric mucosa, atrophy, intestinal metaplasia, and dysplasia are steps conducing to sporadic adenomas of the stomach, usually observed in the older population ${ }^{4}$. Syndromic gastric polyps with these phenotypes have been described and are not the focus of this paper.

The prevalence of gastric polyps around the world is variable, reflecting regional etiologies or associations, and seems to be increasing ${ }^{5-7}$. The two most frequently associated conditions are $H$. pylori eradication treatments and the use of PPls in the treatment of dyspepsia. Both conditions are associated with dynamic variations in the relative prevalence of hyperplastic and FGP5,7.

Since scant data are available on gastric polyps in Mexico ${ }^{8,9}$, our objective was to conduct a new revision of the original slides from all gastric biopsies with a gastric polyp diagnosis from surgical pathology records and describe demographic features of patients and time trends of polyp subtype for the years 1980 , 1990, 2000, 2010, and 2016.

\section{MATERIALS AND METHODS}

We conducted a search in the surgical pathology archives of all gastric biopsies with a morphologic diagnosis performed in the years 1980, 1990, 2000, 2010, and 2016 at Instituto Nacional de la Nutrición Salvador Zubirán, a referral center in Mexico City. Patients with a morphologic diagnosis of any type of gastric polyp were identified, and the slides retrieved from files. The initial diagnosis, gender, age, symptoms, number of lesions, and locations within the stomach were recorded. A blind review of patients' clinical information and morphologic data of the slides stained with hematoxylin and eosin was performed by an experienced pathologist, who used a multi-head microscope (NIKON ECLIPSE 80i). Reviewer diagnosis and the rest of variables were analyzed using descriptive statistics
(SPSS 20). Significance tests were performed using Mann-Kendall test for time trend and $\chi^{2}$ for categorical variables $(p<0.05)$.

Regarding the definition of variables, we considered FGP if microscopy showed cystically dilated glands lined by gastric body type mucosa with parietal cell hyperplasia and minimal inflammation in the stroma. In the case of HP diagnosis, we observed a proliferation of surface foveolar cells lining elongated, distortèd pits, and glands that extend deep into the lamina propria showing edema and inflammation. Moreover, polypoid projection of a dysplastic epithelium associated with atrophy and intestinal metaplasia was established as adenoma $^{10}$.

\section{RESULTS}

A total of 3887 patients underwent gastric biopsies, and 192 epithelial polyps (4.9\%) were identified in the re-revised slides (Fig. 1). The median age of patients with polyps was 58 years (range 19-94 years), and $73 \%$ were female (Fig. 2). A single polyp was föund in $143 / 192(74.4 \%)$, almost $67 \%$ of polyps were located in the oxyntic mucosa and $85 \%$ were associated with dyspepsia. The prevalence of gastric epithelial polyps among patients with gastric biopsies was $0.5 \%, 1.6 \%$, $1.9 \%, 4.6 \%$, and $9.6 \%$ for the years $1980,1990,2000$, 2010 , and 2016, respectively. The rising time trend in these 46 years in the prevalence of epithelial.polyps was $380 \%(p<0.017)$ (Table 1$)$. A relative prevalence of $3.29 \%, 0.97 \%$, and $0.15 \%$ was observed fop FGPs, HPs, and gastric adenoma, respectively (Table 2).

\section{Time trends of gastric polyp subtypes}

HPs were observed in 38/192 (19.8\%) patients. Time trend variations were $0.015,0.004,0.012$, an $\lceil 0.012$ during the years 1980, 1990, 2000, 2010, and 2016 , respectively, a 20\% decrease since 1980 (Eig. 3). To better estimate the percentage of change, we added the years 1980 and 1990; only one polyp was observed between 187 gastric biopsies in 1980 .

According to the histologic diagnosis, FGPs had àglobal frequency of $66.6 \%$ (128/192). They were identified for the first time in the third period of the study, and since then, they constituted $28.6 \%(6 / 21), 66.6 \%$ 
Table 1 . Since 2000 , an increase of $1400 \%$ was identified in the prevalence of fundic gland polyps and a decrease in hyperplastic polyps in the past years of our study.

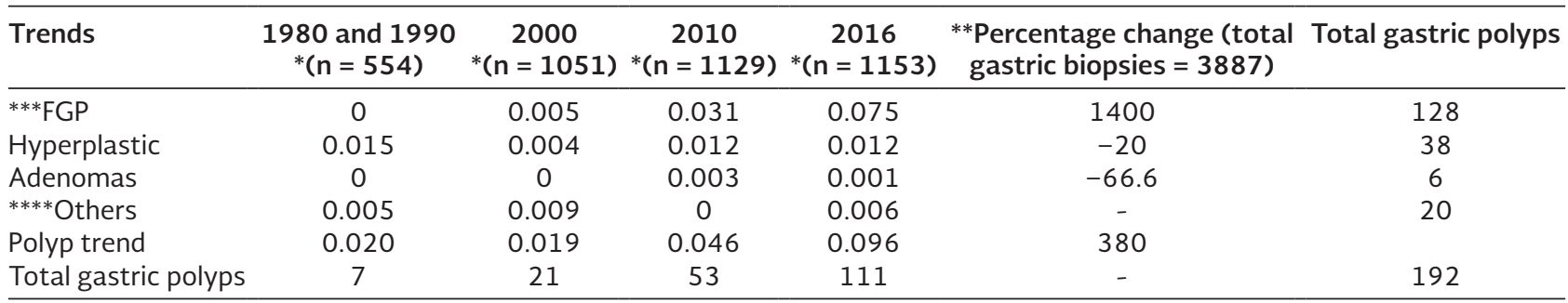

*Total gastric biopsies performed in the year were represented by "n."

**Percentage of change in prevalence was obtained by the formula = (final rate-initial rate/initial rate) 100.

This formula was not available in ****other polyps since there was a value of zero between the years 2000 and 2016

***FGP: Fundic gland polyps.

****Others: Foveolar, hamartomatous, and prolapse type polyps.

Table 2. Polyp subtypes in five different years $(1980,1990$, 2000,2010 , and 2016). A gastric polyp prevalence of $4.9 \%$ was observed with fundic gland polyps explaining most of the cases.

\begin{tabular}{lcc}
\hline Type & $\mathrm{N}$ & Prevalence (\%) \\
\hline *FGP & 128 & 3.29 \\
Hyperplastic & 38 & 0.97 \\
Adenomas & 6 & 0.15 \\
**Others & 20 & 0.51 \\
Total & 192 & 4.93 \\
\hline
\end{tabular}

*FGP: Fundic gland polyps

**Others: foveolar, hamartomatous, and prolapse type polyps

(35/53), and $78.3 \%(87 / 111)$, during the years 2000, 2010, and 2016, respectively. The percentage of change for this lesion since 1990 was $1400 \%$.

Six gastric adenomas were observed ( 4 in 2010 and 2 in 2016), in patients with a median age of 64 years. Less morphologically characterized gastric epithelial polyps were recorded (foveolar and hamartomatous polyps).

\section{Gastric polyp subtypes in relation to gender and age}

Gastric adenomas were mainly observed in older patients. HPs and FGPs were evenly dispersed across all age spectra in this series (Fig. 2). Female-to-male ratio was $3: 1$, without association with the polyp phenotype or year of its morphologic diagnosis (Fig. 2).

Even if the initial endoscopic and histopathologic diagnosis coincided with a gastric polyp diagnosis, the reevaluation of the original slides could not confirm the diagnosis of gastric polyps in 22 ( $10.2 \%$ ) cases, so
Figure 1. There were a total of 3887 gastric biopsies in which 3673 were excluded because there was no gastric polyp diagnosis; 214 reports were re-evaluated from which 22 were excluded since there was no actual polyp. Therefore a total of 192 were included in our study.

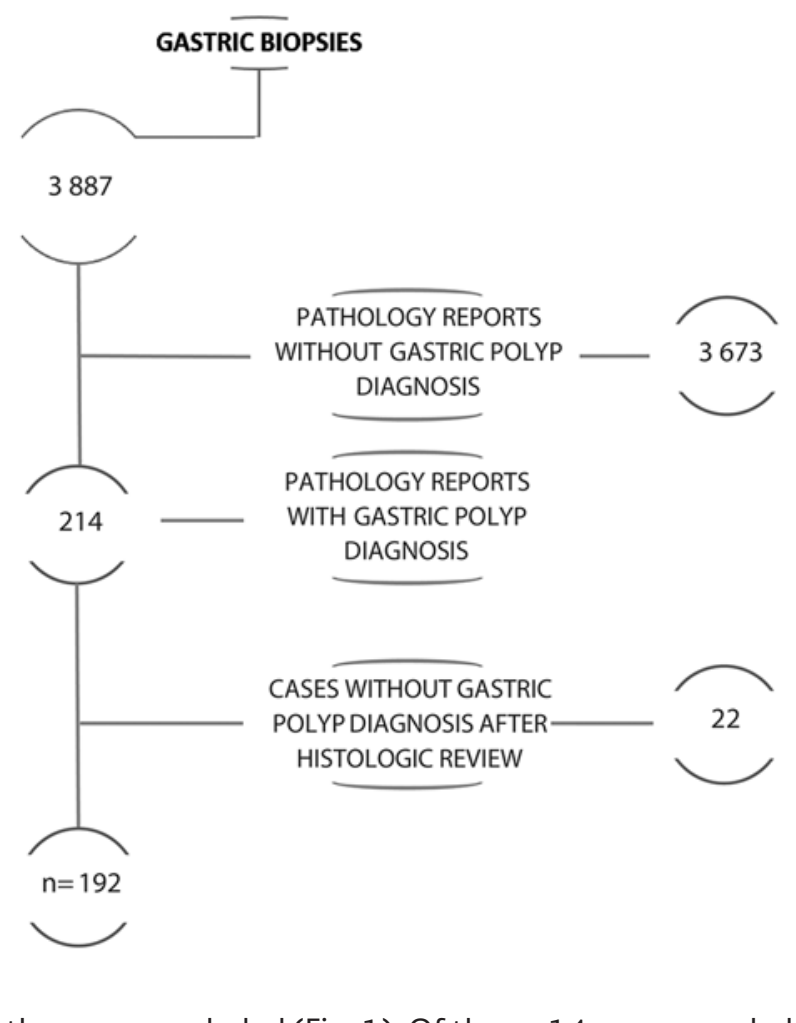

they were excluded (Fig. 1). Of these, 14 corresponded to $H$. pylori-associated gastritis with follicular hyperplasia, 6 to pseudopolyp foveolar hyperplasia; one to an ulcer rim, and another to a mucosal fold. In ànother 22 patients (10.2\%), there was a disagreement with the original polyp phenotype reported. Twelve HPs were reinterpreted as FGPs (8), foveolar polyp (3), and one as a gastric adenoma. Five FGPs were reclassified as hyperplastic (2), foveolar (1), adenoma (1), and 
Figure 2. Gender and age of patients with gastric polyps. A 3:1 female-to-male ratio was observed across all ages. A higher number of patients were older than 58 years $(p<0.001)$.

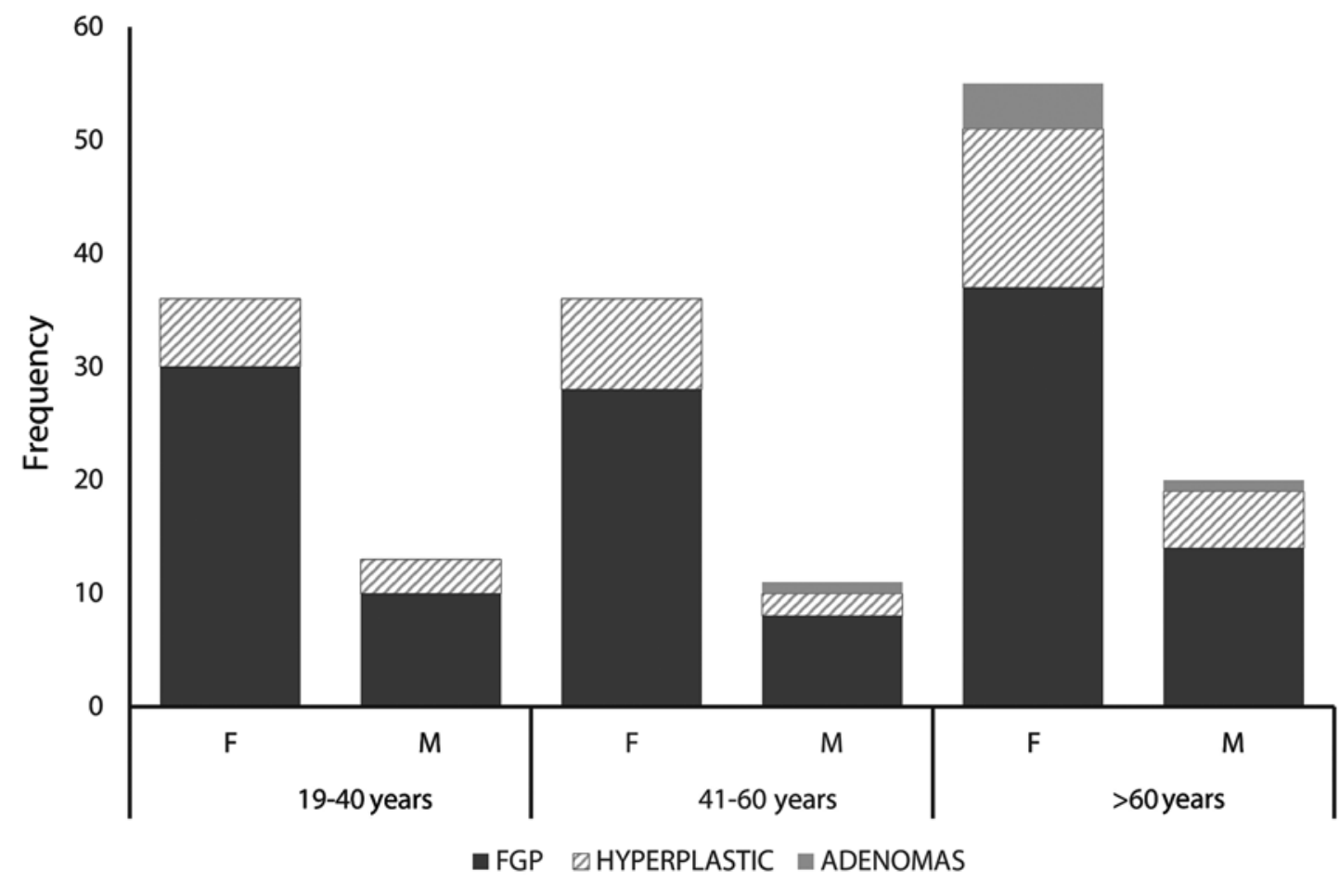

Figure 3. Time trends in gastric polyp subtypes.

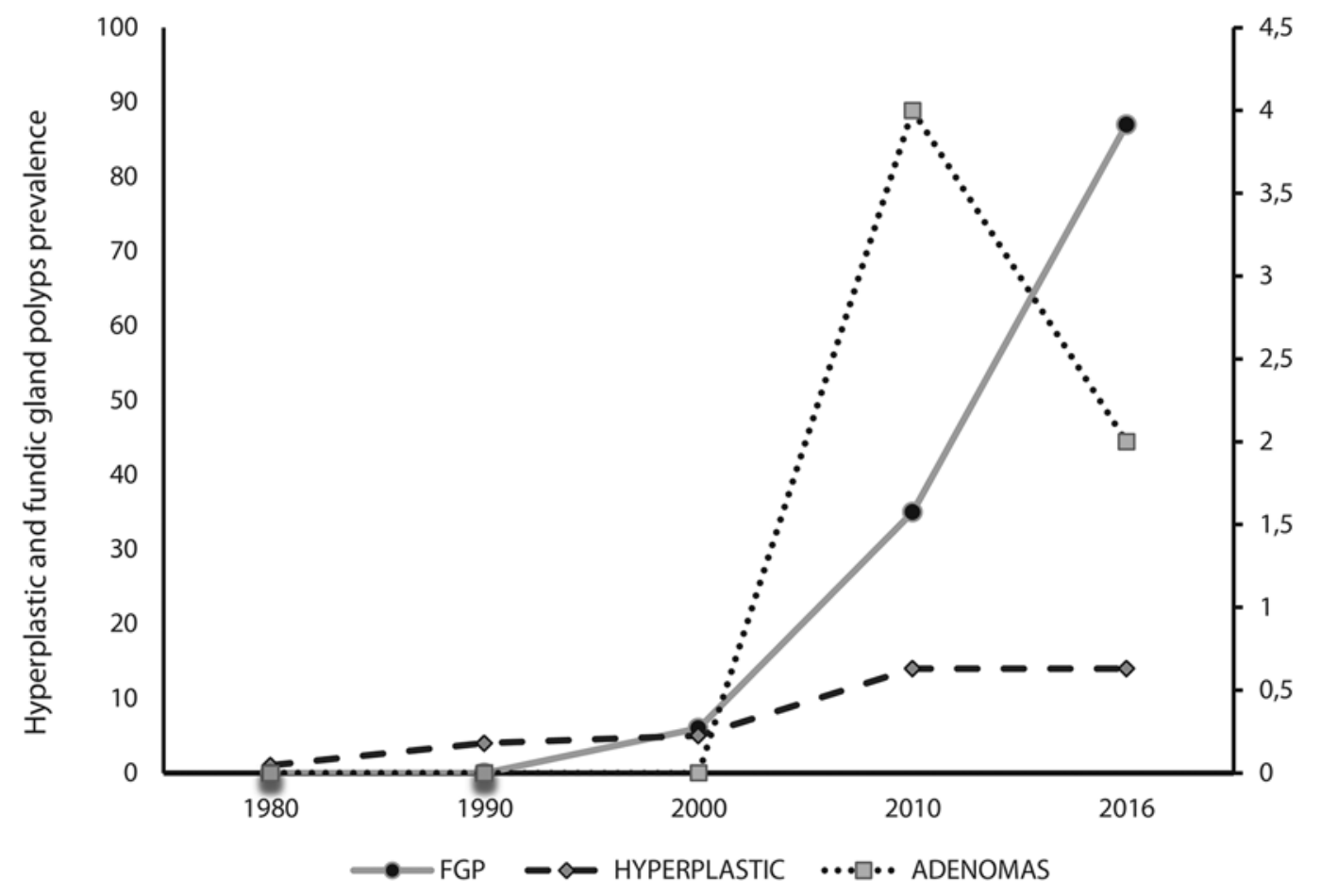

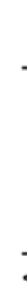


hamartomatous (1) polyps. From 3 lesions considered as adenomas, 2 were foveolar and one a submucosal prolapse gastric polyp.

\section{DISCUSSION}

Even in symptomatic patients submitted to endoscopic sampling of elevated lesions in the gastric mucosa, gastric epithelial polyps are an infrequent finding, observed in $4.93 \%$ of patients in the present study. This prevalence is similar to the $0.58 \%$ and $6.35 \%$ previously reported in different populations ${ }^{11,12}$. However, it is higher than the $0.62 \%$ prevalence reported in a former study describing 100 gastric polyps observed in 15,974 upper endoscopies performed during 9 years (1981-1989) in this same third-level hospi$\mathrm{tal}^{8}$, and closer to the $3.7 \%$ observed during a study from 2011 analyzing 2,913 endoscopies performed in a second-level center in Mexico City (9).

The median age of patients with gastric polyps in this series was 58 years, although a trend for an older age at the time of morphologic diagnosis was observed $(p<$ 0.001 ). A female-to-male ratio of 3:1 was observed for the presence of gastric polyps in biopsies, and this difference was sustained from the year 2000 for the fundic gland and hyperplastic subtypes of polyps. Large $(>1 \mathrm{~cm})$ and pedunculated HPs have a higher risk of malignant transformation, although, in the present study, none of the polyps had epithelial dysplasia ${ }^{13}$. Moreover, adenomas were considered true premalignant conditions of the gastric mucosa, which can lead to carcinoma ${ }^{14}$.

A high proportion of FGPs was identified in women whose age was above the median age of menopause in Mexican women, suggesting hormonal influences on parietal cell dynamics, as we have perceived in animal models ${ }^{15}$. These are questions that already exist on the dynamics of precursors and progression of gastric polyps.

A rising trend in gastric polyp prevalence was identified in this study of symptomatic patients with endoscopic sampling of elevated lesions; there was a $380 \%$ increase during the period of 46 years among patients whose gastroscopies were performed before or after the introduction of PPIs to the market in Europe (1988), United States (1990), and Mexico (1991-1992) ${ }^{14}$. A study from Israel, including patients from 1994 through 2009, and two studies from China analyzing patients from 2000 to 2013 identified increases in gastric polyp prevalence in the PPI era ${ }^{5,7,16}$. Gastric epithelial polyp subtype in the current study has also changed: All the polyps found in 1980 and 1990 were hyperplastic, decreasing by $87 \%$ in frequency in 2016 (percentage of change $-20 \%$ ). On the contrary, FGPs were not found in reviewed piopsies from 1980 and 1990; they were identified for the first time in 2000 and had a constant increase to constitute $78 \%$ of polyps in 2016 (percentage of change $1400 \%$ ). Gastric adenomas were few and without a trend;occurring mainly in older males who had a history of intestinal metaplasia and dysplasia. $H$. pylori eradication and $>12$ months on PPI treatment are considered as explanations for both shifts in some populations ${ }^{3,14}$.

Since the literature regarding the morphologic diaghosis of gastric polyps has been evolving in these $4 \overline{6}$ years, we expected variations from the original surgical pathology report. In 44 biopsies (20.4\%), we found disagreement with the first report, in half of the cases because the phenotype of the lesion was different, and in the other half because the mucosal elevation $\stackrel{\uplus}{\text { was not }}$ secondary to an epithelial polyp. In agreement with previous reports ${ }^{17}$, HPs were more frequently misclasssified. This is a reminder that retrospective studies oncancer or its precursor lesions should not base their ob̄servations only on archival surgical pathology reports?

In summary, this study identifies a rise in gastric epithelial polyps, with FGP as the main phenotype, and a decrease of HP in the past 46 years in a sample of patients undergoing gastric endoscopy in a third-level setting in Mexico City.

\section{ACKNOWLEDGMENTS}

The authors thank Evelyn Matías Benitez, frōm the Benemérita Universidad autónoma de Puebla for searching the 2016 pathology reports.

\section{REFERENCES}

1. Carmack SW, Genta RM, Graham DY, Lauwers GY. Manăgement of gastric polyps: A pathology-based guide for gastroenterologists. Nat Rev Gastroenterol Hepatol 2009; 6:331-41.0

2. Abraham SC, Singh VK, Yardley JH, Wu TT. Hyperplastic polyps of the stomach: Associations with histologic patterns of gastritis and gastric atrophy. Am J Surg Pathol 2001; 25:500-7.

3. Jalving M, Koornstra J], Wesseling J, Boezens HM, De Jong S, Kleibeuker $\mathrm{JH}$. Increased risk of fundic gland polyps during 
long-term proton pump inhibitor therapy. Aliment Pharmacol Ther 2006; 24:1341-8.

4. Palacios Salas F, Velarde F, Palomino Portilla E. Gastric polyps and histological changes in surrounding mucosa. Rev Gastroenterol Peru 2003; 23:245-53.

5. Peretz A, Fuchs T, Livovsky DM, Turvall E, Pappo O, Ackerman $Z$. The changing histological pattern of gastric polyps in an ethnically heterogeneous population. Scand J Gastroenterol 2012; 47:907-13.

6. Tran-Duy A, Spaetgens B, Hoes AW, de Wit NJ, Stehouwer CDA. Use of proton pump inhibitors and risk of fundic gland polyps and gastric cancer: Systematic review and meta-analysis. Clin Gastroenterol Hepatol 2016; 14:1706-19.

7. Cao H, Wang B, Zhang Z, Zhang H, Qu R. Distribution trends of gastric polyps: An endoscopy database analysis of 24,121 northern Chinese patients. J Gastroenterol Hepatol 2012; 27:1175-80.

8. Gallo Reynoso S, Rodríguez Hernández H, Elizondo Rivera J. Gastric polyps. Experience at the Department of Endoscopy of the Salvador Zubirán National Institute of Nutrition. Rev Gastroenterol Mex 1990; 55:51-4.

9. Montenegro-Molina W, Zamorano-Orozco Y, MartínezGarcía CL. Prevalence of gastric polyps and histological correlation at the "Dr. Carlos Mac Gregor Sánchez Navarro" Hospital. Endoscopia 2011; 23:23-8.
10. Goddard AF, Badreldin R, Pritchard DM, Walker MM, Warren B. The management of gastric polyp. Gut. 2010; 59: 1270-6.

11. Carmack SW, Genta RM, Schuler CM, Saboorian MH. Ihe current spectrum of gastric polyps: A 1-year national study of over 120,000 patients. Am J Gastroenterol 2009; 104:1524-32.

12. Morais DJ, Yamanaka A, Zeitune JM, Andreollo NA. Gastric polyps: A retrospective analysis of 26,000 digestive endoscopies. Arq Gastroenterol 2007; 44:14-7.

13. Ahn JY, Son DH, Choi KD, Roh J, Lim H, Choi KS, et al. Neoplasms arising in large gastric hyperplastic polyps: Endoscopic and pathologic features. Gastrointest Endosc 2014; 80:1005-13.

14. Sonnenberg A, Genta RM. Prevalence of benign gastric polyps in a large pathology database. Dig Liver Dis 2015; 47.164-9.

15. Saqui-Salces M, Neri-Gómez T, Gamboa-DomínguezAA, RuizPalacios G, Camacho-Arroyo I. Estrogen and progesterone receptor isoforms expression in the stomach of Mongdian gerbils. World J Gastroenterol 2008; 14:5701-6.

16. Fan NN, Yang J, Sun G, Lu ZS, Hu E, Wang XD, et al. Changes in the spectrum of gastric polyps in the Chinese population. World J Gastroenterol 2015; 21:9758-64.

17. Gonzalez-Obeso E, Fujita H, Deshpande V, Ogawa F, Lisogrsky M, Genevay M, et al. Gastric hyperplastic polyps: A heterogeneous clinicopathologic group including a distinct subset best categorized as mucosal prolapse polyp. Am J Surg Pathol 2011; 35:670-7. 